\title{
Modeling of the Transitional Pressure Drop of Fibrous Filter Media Loaded with Oil-coated Particles
}

\author{
Ta-Chih Hsiao $^{1 *}$, Da-Ren Chen ${ }^{2}$ \\ ${ }^{1}$ Graduate Institute of Environmental Engineering, National Taiwan University, Taipei 10617, Taiwan \\ ${ }^{2}$ Department of Mechanical and Nuclear Engineering, Virginia Commonwealth University, Richmond, VA 23284, USA
}

\begin{abstract}
The pressure drop of glass-fiber and cellulose filter media loaded with oil-coated particles was investigated. The focus of this study was to develop a model describing the pressure drop of fibrous filter media under the above particle loading condition. A set of experimental data collected during previous work was used for this modelling. For the cases where the coated particles possessed an oil volumetric percentage below 50\%, the filter was divided into two layers: One layer collected all test particles while the other layer remained clean, and the pressure drop of the first layer was estimated using a modified Bergman model, whereas that of the second was calculated with the Davies equation. The total filter pressure drop is the summation of the layers' pressure drops. For the cases where the coated particles possessed an oil volumetric percentage above $50 \%$, a power-law equation with two parameters (viz., the exponent, $n$, and the critical volume, $V_{c r}$ ) was applied to fit the experimental data. The correlations of the above parameters with the solid-core diameter fraction $(X)$ of the particles and the viscosity of the coating oil were calculated for the glass-fiber and cellulose filter media.
\end{abstract}

Keywords: Fibrous filter; Aerosol filtration; Loading behavior; Oil-coated particle.

\section{INTRODUCTION}

Filtration of aerosol particles is a dynamic process (Bao et al., 2015; Choi et al., 2017; Sachinidou et al., 2017). The filter loading process is typically recorded as the filter pressure drop in the function of time (when the size distribution of sampled particles is known) or particle mass/volume collected by filter media of unit area. As particles are trapped in filter media or collected on the media surface, the particle collection efficiency of filter media often continually improves and the pressure drop across the media monotonically increases (Park et al., 2012). While the increase of particle collection efficiency of filtration media is a benefit, the increase in the filter pressure drop is undesirable. It is because the increase of filter pressure drop often results either in the reduction of filtration velocity or the increase in the load of air movers (consequently reducing the lifetime of air movers).

Various models have been proposed to estimate the time evolution of filter pressure drop when loaded with particles (Kanaoka and Hiragi, 1990; Endo et al., 1998; Thomas et al., 1999; Leung and Hung, 2008; Saleh and Tafreshi, 2014).

\footnotetext{
* Corresponding author.

Tel.: (+886) 2-3366-4401; Fax: (+886) 2-2392-8830

E-mail address: tchsiao@ntu.edu.tw
}

The modelling to predict the time evolution of filter pressure drop when continuously loaded with particles, especially in the depth and transitional filtration phases, remains a challenging task. To understand the transitional-loading behavior of filter media, the effect of various factors (e.g., filter medium, physical properties of particles, filtration face velocity, and relative humidity) have been extensively studied. A majority of previous studies have been focused on the loading of filters with only solid particles (Japuntich et al., 1994; Veerapaneni and Wiesner, 1997; Endo et al., 1998; Chen et al., 2001; Thomas et al., 2001; Song et al., 2006; Müller et al., 2010; Joubert et al., 2011; Saleh and Vahedi Tafreshi, 2015) and few on the filtration of liquid particles (Liew and Conder, 1985; Agranovski and Braddock, 1998a, b; Zhang et al., 2017). For liquid particle loading, the droplet migration on fibers in filter media is a very complex process and it is affected by a variety of factors, such as the operational flow condition and the physical property of loaded liquid (liquid viscosity and surface tension) (Chang et al., 2016).

In addition to pure solid and liquid particles, particles with a mixed phase (in the form of solid particles coated or mixed with liquids) often exist in the real world. These multi-phased particles are typically called "greasy oils." Examples of such greasy particles or oil-coated particles are those emitted from internal combustion engines or generated in vehicle crankcases, and those produced during grinding and milling operations (with metal working fluid) 
(Hsiao and Chen, 2015; Wei et al., 2017). Due to their physical property difference, the pressure drop evolution curve of a fibrous filter media loaded with oil-coated particles is very different from that of a medium loaded with either pure solid or pure liquid particles. As a result, the existing pressure drop models for filter media loaded with pure solid or liquid particles cannot be directly applied to describe the filter pressure drop evolution when the filter media is loaded with oil-coated particles. One possible method to holistically modeling the loading behavior of a fibrous filter medium loaded with oil-coated particles is to integrate and modify the pressure drop models for filter media loaded with pure liquid or solid particles. The objective of this study is thus to develop a model for predicting the pressure drop evolution of fibrous filter media when loaded with oil-coated particles.

\section{PRESSURE DROP OF AN AEROSOL FILTER}

Prior to the presentation of our modeling work on the pressure drop of filter media loaded with oil-coated particles, the pressure drop models of clean filter media and loaded filters are briefly reviewed.

\section{Clean Filter}

The basic concept to estimate the clean filter pressure drop is on the force balance. It is assumed that the overall pressure drop results from the summation of drag forces acting on all the fibers in filter media of a unit area. At a low Reynolds number (i.e., $\rho U d_{f} / \mu<<1$ ), the drag force on a single fiber can be approximated by the product of the flow velocity, viscosity, and drag coefficient $(f)$. The pressure drop of a clean filter, $\Delta P_{0}$, can then be formulated as:

$\Delta P_{0}=F_{D} \cdot L_{f}=\mu U f \cdot L_{f}$

where $F_{D}$ is the fiber drag force per unit of fiber length; $L_{f}$ is the total fiber length per unit area of filter media (which can be derived from the packing density, $\alpha_{f}$, and thickness of the filter, $Z$, when a single fiber diameter, $d_{f}$, is given).

$L_{f}=4 \alpha_{f} Z / \pi d_{f}^{2}$
The accuracy of the above filter pressure drop modeling is highly dependent on the $f$. Four general expressions of $f$ for estimating the pressure drop across a clean filter are summarized in Table 1.

The expressions for $f$ proposed by Happel (1959), Kuwabara (1959), and Fuchs and Stechkina (1963) were all derived theoretically by solving the flow field in a system of parallel and circular cylinders at a low Reynolds number with different sets of boundary conditions. Happel assumed that the normal velocity and tangential stress at the cylindrical surface are 0 , whereas Kuwabara assumed that the normal velocity and vorticity are 0 . However, experimental results generally indicate that the accuracy of these expressions in predicting filter pressure drops is less favorable than the empirical expression proposed by Davies (1953). The discrepancy between theoretical prediction and experimental measurements is possibly due to the random orientation of fiber cylinders and the flow interference among them in experiments (Yamada et al., 2011; Zhou et al., 2017). When Davies' $f$ expression is chosen and combined with Eq. (2), the pressure drop of a clean filter can be expressed as:

$\Delta P_{0}=\mu U Z \cdot \frac{64 \alpha_{f}^{1.5}\left(1+56 \alpha_{f}^{3}\right)}{d_{f}^{2}}$ for $0.006<\alpha_{f}<0.5$, and

$\Delta P_{0}=\mu U Z \cdot \frac{64 \alpha_{f}^{1.5}}{d_{f}^{2}}$ for $\alpha_{f}<0.006$

The above equations are semi-empirical and do not take into consideration the fiber size variation in filter matrix. A thorough survey by Jackson and James (1986) indicated that the prediction begins to deviate from the experimental measurements when the packing density is less than 0.001 .

\section{Filter Loaded with Solid Particles}

Kanaoka and Hiragi (1990) developed an early theoretical model of the pressure drop across a dust-loaded filter, $\Delta P_{l}$. They also employed the concept of summing the total drag forces acting on the dust-loaded fibers, $F_{l}$, which was

Table 1. Drag coefficients, $f$, for an individual cylinder fiber.

\begin{tabular}{ll}
\hline Expression of $f$ & Reference \\
\hline$f=8 \pi \frac{1}{-\ln \alpha_{f}-\left(\left(1-\alpha_{f}^{2}\right) /\left(1+\alpha_{f}^{2}\right)\right)}$ & Happel (1959) \\
$f=8 \pi \frac{1}{-\ln \alpha_{f}-2 \alpha_{f}-0.5 \alpha_{f}^{2}-1.5}$ & Kuwabara (1959) \\
$f=8 \pi \frac{1}{-\ln \alpha_{f}-1.5}$ & Fuchs and Stechkina (1963) \\
$f=16 \pi \alpha_{f}^{0.5}\left(1+56 \alpha_{f}^{3}\right)$ for $0.006<\alpha_{f}<0.5$ & Davies (1953) \\
$f=16 \pi \alpha_{f}^{0.5}$ for $\alpha_{f}<0.006$ & \\
\hline
\end{tabular}


evaluated in the manner of Newton's resistance law. The diameter of a dust-loaded fiber $\left(d_{f m}\right)$ was chosen as a representative parameter of dendritic structure.

$$
\begin{aligned}
& \Delta P_{l}=L_{f} \int_{0}^{Z} F_{l} d z, \text { and } \\
& F_{l}=C_{d m} d_{f m} \cdot \frac{\rho U^{2}}{2} .
\end{aligned}
$$

The drag coefficient of dust-loaded fibers per unit filtration area $\left(C_{d m}\right)$ and the diameter of a dust-loaded fiber $\left(d_{f m}\right)$ are two critical parameters in this model. They were correlated with the filtration condition and collection mechanism along with the dimensionless accumulated particle volume, $V_{\text {ac }}$, defined as the ratio of loaded particle mass to particle density and filter packing density per unit filter volume. Kanaoka and Hiragi (1990) further classified the rate of increase of $d_{f m}$ into three stages: no growth at low $V_{\text {ac }}$, rapid growth at intermediate $V_{\text {ac }}$, and dampened growth at high $V_{\text {ac }}$. This model has been claimed as applicable to predict the pressure drop of a dust-loaded filter under any filtration conditions. However, the value of $d_{f m}$ and $C_{d m}$ are difficult to be estimated theoretically. They were given by empirical fitting with the accumulated volume of captured particles and cannot be determined without performing experiments.

Instead of evaluating the drag forces of particle dendrites formed by deposited particles, Bergman et al. (1978) considered the particle dendrites as newly formed fibers and modified the Davies equation to include an additional pressure drop due to these newly formed fibers. In addition, to correct the interference between the dendrites and filter fibers, they increased the fiber and dendrite volume fraction by the factors $\left(L_{f}+L_{p}\right) / L_{f}$ and $\left(L_{f}+L_{p}\right) / L_{p}$ respectively:

$$
\begin{aligned}
& \Delta P_{l}= \\
& 16 \pi \mu U \cdot\left[\left(\alpha_{f} \cdot \frac{L_{f}+L_{p}}{L_{f}}\right)^{0.5} \cdot L_{f}+\left(\alpha_{p} \cdot \frac{L_{f}+L_{p}}{L_{p}}\right)^{0.5} \cdot L_{p}\right] .
\end{aligned}
$$

where $L_{p}$ can be evaluated as follows:

$$
L_{p}=\frac{4 \alpha_{p} Z}{\pi d_{p}^{2}}
$$

By combining with Eq. (6), the expression for the pressure drop across a loaded filter can be derived:

$$
\Delta P_{l}=64 \mu U Z \cdot\left(\frac{\alpha_{f}}{d_{f}}+\frac{\alpha_{p}}{d_{p}}\right) \cdot\left(\frac{\alpha_{f}}{d_{f}^{2}}+\frac{\alpha_{p}}{d_{p}^{2}}\right)^{0.5} .
$$

The general criticism of the model of Bergman et al. (1978) is about the assumption of a uniform distribution of deposited particles on the filter matrix. Hence, Thomas et al. $(1999,2001)$ proposed to divide the entire filter into multiple layers and to evaluate the layers' collection efficiency and pressure drops at every time step based on the information obtained from prior layers. Although such a method is ultimately closer to realistic conditions, the calculation is cumbersome and the result can depend on the number of layers, which is arbitrarily determined.

\section{Filter Loaded with Liquid Particles}

For a fibrous filter loaded with liquid particles, only a few models have been established. These models are primarily applicable to filters under steady-state saturation conditions. Liew and Conder (1985) performed various tests on filters with mean fiber diameters of $4,8,12$, and $22 \mu \mathrm{m}$ and developed an empirical equation to predict the filter pressure drop at the final steady-state stage, $\Delta P_{s}$ :

$$
\frac{\Delta P_{s}}{\Delta P_{0}}=\left[1.09 \cdot\left(\alpha_{e} \frac{Z}{d_{f}}\right)^{-0.561} \cdot\left(\frac{U \mu}{\gamma_{L V} \cdot \cos \theta}\right)^{-0.477}\right]
$$

where $\gamma_{L V}$ is the liquid surface tension and $\theta$ is the contact angle between a deposited droplet and a fiber.

Raynor and Leith (2000) provided another empirical expression for $\Delta P_{s}$, which is correlated with the steady-state saturation ratio, $S_{e}$, and the steady-state packing density, $\alpha_{e}$ :

$\ln \left(\frac{\Delta P_{s}}{\Delta P_{0}}\right)=\frac{S_{e}^{0.91 \pm 0.06}}{\alpha^{0.69 \pm 0.06}} e^{-1.21 \pm 0.24}$

They also constructed an empirical expression for $S_{e}$ against the dimensionless numbers $(\mathrm{Ca}, \mathrm{Bo}, \mathrm{Dr})$ using the commercial statistical software SAA/STAT:

$\begin{aligned} S_{e}= & \alpha^{0.39 \pm 0.09} /\left(\mathrm{Bo}^{[(0.47 \pm 0.06)+(0.24 \pm 0.07) \cdot \ln \mathrm{Bo}]} \cdot \mathrm{Ca}^{(0.11 \pm 0.04)}\right. \\ & \left.\cdot \mathrm{e}^{[(-0.04 \pm 0.36)+(6.6 \pm 0.15) \times 105 \cdot \mathrm{Dr}]}\right)\end{aligned}$

where Bo is the bond number $\left(\rho g d_{f}^{2} / \gamma_{L V} \times 10^{5}\right), \mathrm{Ca}$ is the capillary number $\left(\mu \mathrm{U} / \gamma_{\mathrm{LV}} \times 10^{5}\right)$, and $\mathrm{Dr}$ is the nondimensional drainage rate.

Both models are purely empirical and only applicable for certain operational flow ranges. For example, the model of Liew and Conder (1985) requires the filter packing density to be larger than 0.02 , whereas that of Raynor and Leith (2000) is only feasible for a filter thickness of less than $0.88 \mathrm{~cm}$. Moreover, these two models only predict the steady-state pressure drop of a liquid-loaded filter.

Frising et al. (2005) attempted to establish a pressure drop model for different filtration and loading stages based on the Davies equation. To imitate the gradual clogging of a filter, they characterized the entire loading process into four stages and divided the filter media into $n_{p}$ layers with thickness $d Z$ (i.e., $Z / n_{p}$ ). In the first stage, the loaded liquid particles are assumed to perfectly coat the filter fibers and form liquid films on or around individual fibers. Therefore, 
the fiber diameter, $d_{f}$, and packing density, $\alpha_{f}$, are replaced with the "coated" fiber diameter, $d_{f, w}$, and new packing density $\left(\alpha_{f}+\alpha_{l}\right)$, which includes the loaded droplets.

$$
\begin{aligned}
& d \Delta p= \\
& 64 \mu U d Z \cdot \frac{\left(\alpha_{f}+\alpha_{l}\right) \cdot\left(\alpha_{f}+\alpha_{l}\right)^{0.5}}{d_{f, w}^{2}} \cdot\left(1+16\left(\alpha_{f}+\alpha_{l}\right)^{2.5}\right), \text { and } \\
& d_{f, w}=d_{f} \cdot \sqrt{\frac{\alpha_{f}+\alpha_{l}}{\alpha_{f}}} .
\end{aligned}
$$

The second stage is defined as the formation of the liquid bridge and liquid film at the fiber intersection, and the diameter of a "coated" fiber remains constant in this stage. Because the air flow is greatly influenced by the presence of liquid bridge and films, the air velocity must be modified. The pressure drop equation for the second stage is given as:

$$
\begin{aligned}
d \Delta p & =64 \mu U d Z \cdot \frac{\left(\alpha_{f}+\alpha_{\text {tube }}\right) \cdot\left(\alpha_{f}+\alpha_{l}\right)^{0.5}}{d_{f, w}^{2}} \\
& \cdot\left(1+16\left(\alpha_{f}+\alpha_{l}\right)^{2.5}\right) \cdot \frac{U}{\left(1-\alpha_{l}+\alpha_{\text {tube }}\right)}
\end{aligned}
$$

where $\alpha_{\text {tube }}$ is the maximal packing density resulted by the liquid coating on the filter fibers. However, $\alpha_{\text {tube }}$ cannot be determined either theoretically or experimentally. In the work of Frising et al. (2005), the above value was determined empirically to optimize the prediction of the proposed model. In the third stage, the liquid packing density reaches the maximum, and the liquid migration between filter layers begins. The pressure drop is considered constant at this stage. Once the liquid begins to drain out of the filter, the loading process enters the fourth stage, in which:

$$
\begin{aligned}
d \Delta p & =64 \mu U d Z \cdot \frac{\left(\alpha_{f}+\alpha_{\text {tube }}\right) \cdot\left(\alpha_{f}+\alpha_{f i m m}\right)^{0.5}}{d_{f, w}^{2}} \\
& \cdot\left(1+16\left(\alpha_{f}+\alpha_{f i l m}\right)^{2.5}\right) \cdot \frac{U}{\left(1-\alpha_{l}+\alpha_{\text {tube }}\right)},
\end{aligned}
$$

where $\alpha_{f i l m}$ is the maximal liquid packing density and can be estimated by weighing the test filter before and after experiments. Frising et al. (2005) reported reasonable agreement between their experimental results and the predictions of their model and stated that the model requires only two parameters - $\alpha_{\text {tube }}$ and $\alpha_{\text {film }}$. However, Mullins and Kasper (2006) argued that the assumption of perfect liquid coating of filter fibers does not always hold. For liquids with high surface tension, deposited droplets create liquid beads, rather than forming a film, on the fibers in filter media (Brown, 1993).

\section{MODELING THE PRESSURE DROP OF FILTERS LOADED WITH OIL-COATED PARTICLES}

In our previous experimental study (Hsiao and Chen, 2015), the co-solvent method was applied for generating oil-coated particles. A master solution of oil-coated particles was prepared by mixing two parent solutions, coating oil dissolved in 2-propanol and potassium chloride $(\mathrm{KCl})$ dissolved in DI water, using a volume ratio of 1:1. Four coating oils (DEHS, light mineral oil, castor oil, and glycerol) were selected and tested. Accordingly, the loading curve of a filter loaded with oil-coated particles changes from that of a filter loaded with pure solid particles to that of a filter loaded with pure liquid droplets as the thickness of the liquid coating increases. When the liquid volumetric percentage is less than $50 \%$, the pressure drops of glass-fiber filters and cellulose filters are primarily caused by the solid fraction of the loaded oil-coated particles. Moreover, the loading curve for a low-surface-tension liquid becomes independent of the liquid's viscosity because the total loaded volume is multiplied by the solid volumetric percentage. In other words, it was found that all the curves are more closely positioned for both the glass fiber and the cellulose filter media when the pressure drop evolution curves were re-plotted using the solid core particle volume per unit filter area as the abscissa (Hsiao and Chen, 2015). Thus, the model for a filter loaded with oil-coated particles having a liquid volumetric percentage of less than $50 \%$ can be established based on the modified Bergman method. Because the impaction is the major filtration mechanism in filter media used in our previous testing, the front layer was assumed to collect all particles, and the rear layer was assumed to remain as a clean filter. Thus, the total pressure drop, $\Delta P$, of a filter medium is then the linear sum of the pressure drops across these two layers, and Eqs. (3) and (8) were used to estimate the values.

$\Delta P=\Delta P_{0}^{\prime}+\Delta P_{l}^{\prime}$

The thickness of the front layer $\left(Z_{f L}\right)$ is the critical parameter for the model's predictions, and was estimated from scanning electron microscopy (SEM) images of the loaded filters. The depths of the front layers used for the glass-fiber filter and cellulose filter were $190 \mu \mathrm{m}$ and 55 $\mu \mathrm{m}$, respectively. The other filter characteristics required for the model are listed in Table 2, and $\alpha_{p}$ was calculated based on the loaded particle volume.

As shown in Fig. 1, the curves predicted by the modified Bergman model demonstrated reasonable agreement with the experimental results for both the glass-fiber filter and cellulose filter up to the pressure drop ratio (defined as the ratio of the loaded filter pressure drop to the clean filter pressure drop) of 4 . By recalculating the loaded particle volume, the model can be extended to predict the loading behavior for oil-coated particles, which have a coating with a maximum of $50 \%$ liquid by volume.

For the loading case with the liquid volumetric percentage higher than $50 \%$, the transitional behavior is much more dynamic and strongly dependent on the liquid's properties 
Table 2. Characteristics of the test filter media.

\begin{tabular}{llll}
\hline Test Filter Medium & Filter Thickness $(\mathrm{mm})$ & Basic Weight $\left(\mathrm{kg} \mathrm{m}^{-2}\right)$ & Porosity $(-)$ \\
\hline Cellulose Filter & 0.71 & 0.136 & 0.877 \\
Glass-fiber Filter & 0.45 & 0.112 & 0.904 \\
\hline
\end{tabular}
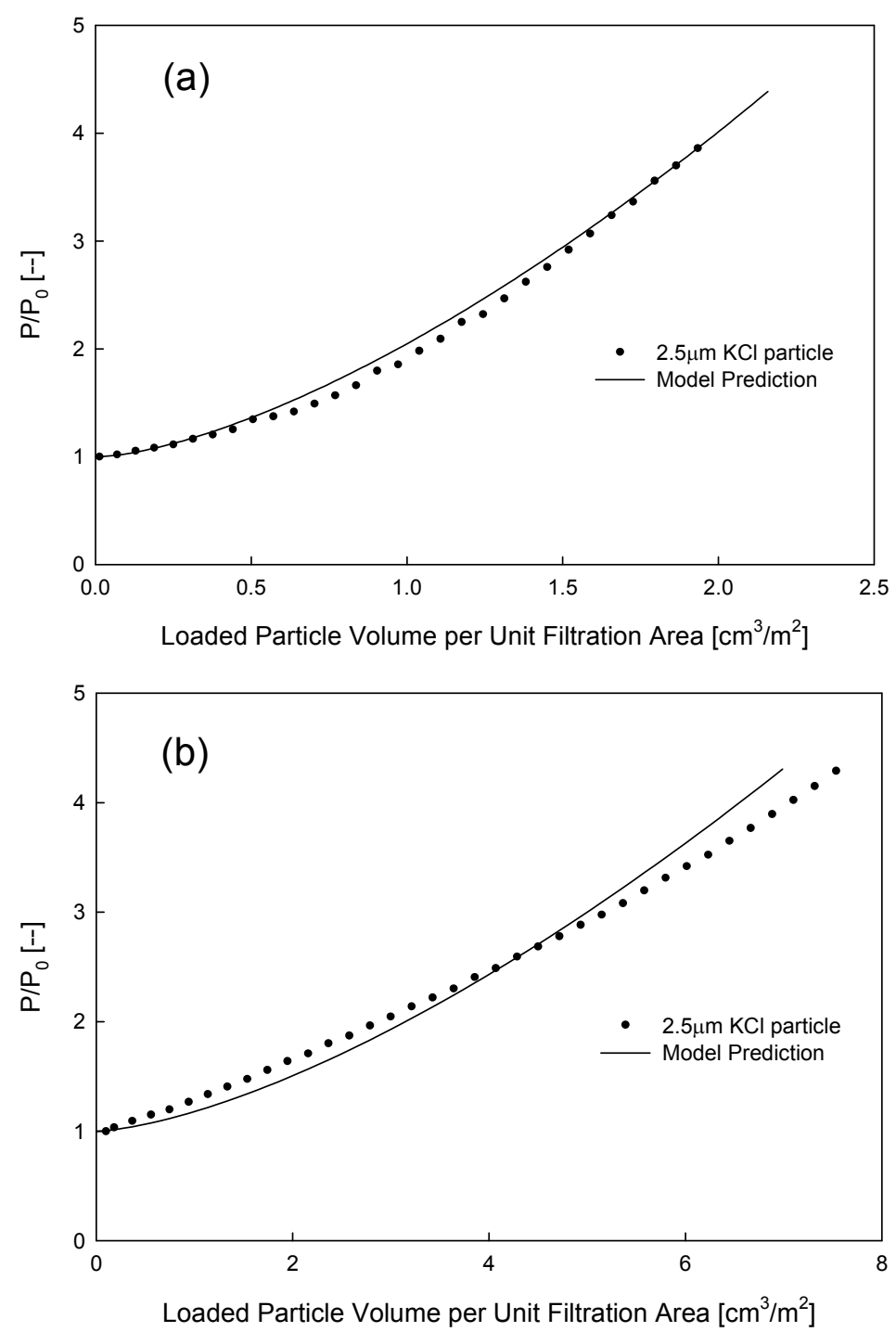

Fig. 1. Experimental data and model prediction for (a) the glass-fiber filter loaded with $2.5-\mu \mathrm{m}$ solid $\mathrm{KCl}$ particles and (b) the cellulose filter loaded with $2.5-\mu \mathrm{m}$ solid $\mathrm{KCl}$ particles.

(both liquid viscosity and surface tension) as well as the filter medium's characteristics (i.e., either absorptive or non-absorptive). No theoretical/empirical model existed to describe the transitional behavior in the cases. We thus proposed the power-law equation with two parameters, i.e., the exponent, $n$, and critical volume, $V_{c r}$, to fit the experimental data. In the proposed equation, $n$ controls the curve growing slope, and $V_{c r}$ controls the curve horizontal scale:

$$
\frac{\Delta P}{\Delta P_{0}}=1+\left(\frac{V}{V_{c r}}\right)^{n}
$$

where $\Delta P_{0}$ is the initial pressure drop of filter, and $V$ is loaded particle volume per unit filtration area.

A similar approach was employed by Hermans and Bredée (1936) and Gonsalves (1950) in hydrosol filtration and was later applied to aerosol filtration by Emi et al. (1982). Although a satisfactory result was reported by Emi et al. (1982). for collection efficiency data, its correlation with pressure drop was not evident. In the present study, however, the loading curves for different oil-coated particles are well fitted by Eq. (17) for at least up to 4 times the initial filter pressure drop, shown in Figs. 2(a) and 2(b).

To establish an empirical model for calculating the filter pressure drop when loaded with oil-coated particles, the 

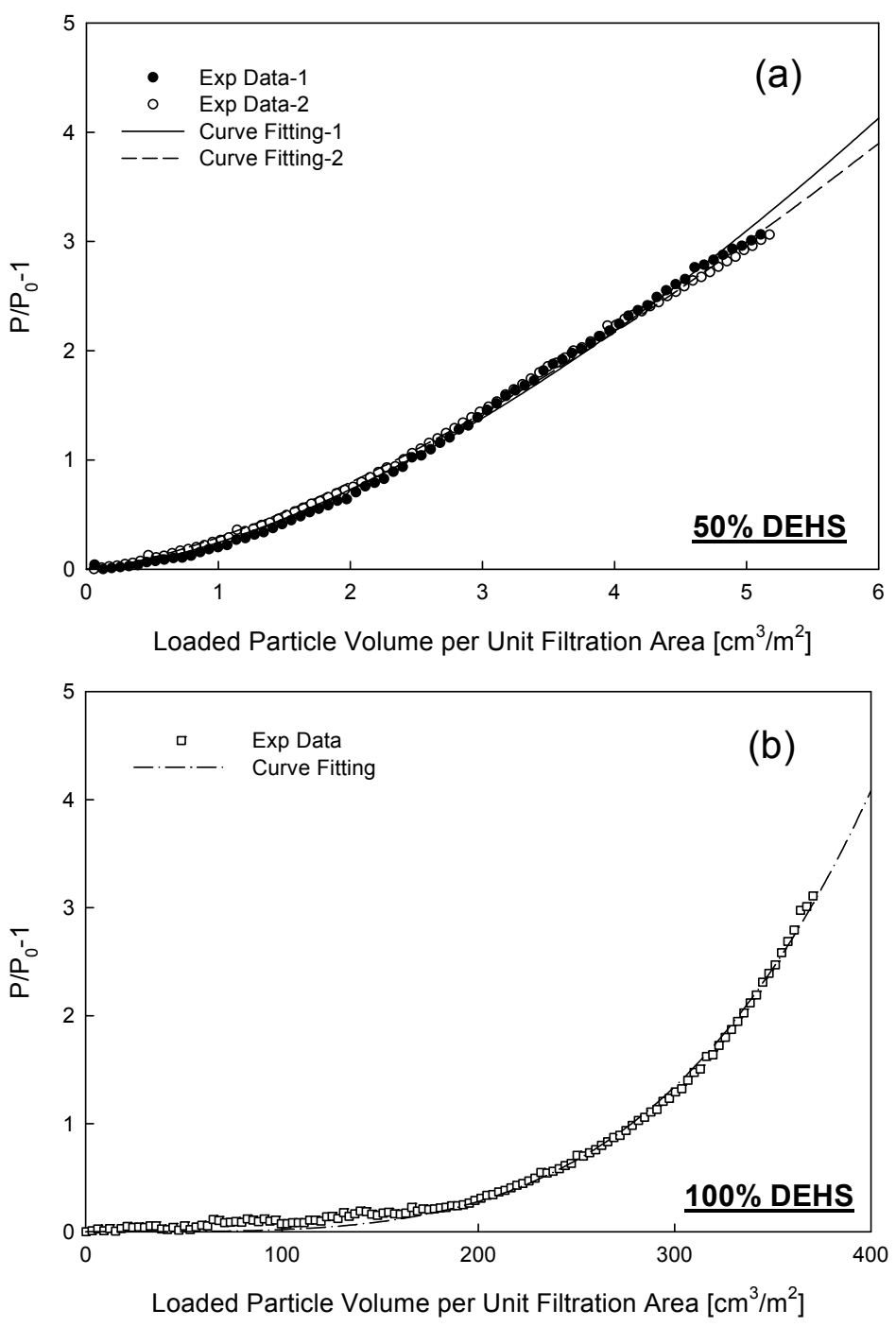

Fig. 2. Experimental data and fitting results for (a) the glass-fiber filter loaded with $2.5-\mu \mathrm{m} 50 \%$ DEHS-coated particles and (b) the glass-fiber filter loaded with $2.5-\mu \mathrm{m} 100 \%$ DEHS particles.

data reported in Hsiao and Chen (2015) were used. The detailed information about different oil-coated particles and the corresponding loading behaviors can be found there. In this study, the data for loading with glycerol-coated particles were not included in this fitting. It is because the surface tension and viscosity of glycerol are very different from those of other coating liquids. Moreover, the effects of surface tension and viscosity on filter pressure drop were difficult to qualitatively differentiate based on the data previously collected. Therefore, the parameters, $V_{c r}$ and $n$, were only correlated to the viscosity of coating liquids in this work.

To illustrate the fitting result, both $V_{c r}$ and $n$ parameters are plotted as the fraction of solid core in the overall particle diameter $(X)$, instead of the volumetric percentage of coating liquid. The solid-core diameter fractions in oilcoated particles corresponding to $0 \%, 20 \%, 50 \%, 88 \%$, and $100 \%$ of liquid-volumetric percentage were $1.0,0.93$, 0.79, 0.49, and 0. As shown in Figs. 3 and 4 (for glassfiber and cellulose filter media, respectively), the values of $V_{c r}$ and $n$ became invariant when the solid core diameter fraction was greater than 0.79 (for coating liquids with similar surface tension). The effect of liquid viscosity on the fitted $V_{c r}$ and $n$ parameters was negligible. However, below the above critical diameter fraction, the $V_{c r}$ and/or $n$ values started to vary. The observed variation was then correlated with the viscosity of coating liquids.

For the glass-fiber filter, as the solid core diameter fraction approached 0 , the value of $V_{c r}$ for different coating liquids approached to the same value (Fig. 3). It implied that the surface tension effect dominated the viscosity effect when loading pure liquid droplets (mist) on a glass-fiber filter medium. Differently, the variation in the power $n$ for different coating liquids was minor for glass-fiber filter media (Fig. 4). The parameter of $n$ was approximately estimated by a polynomial of the third order:

$n=1.059+1.243 X-1.516 X^{2}+0.468 X^{3}$

where $X$ is the diameter fraction of solid core in oil-coated particles. 


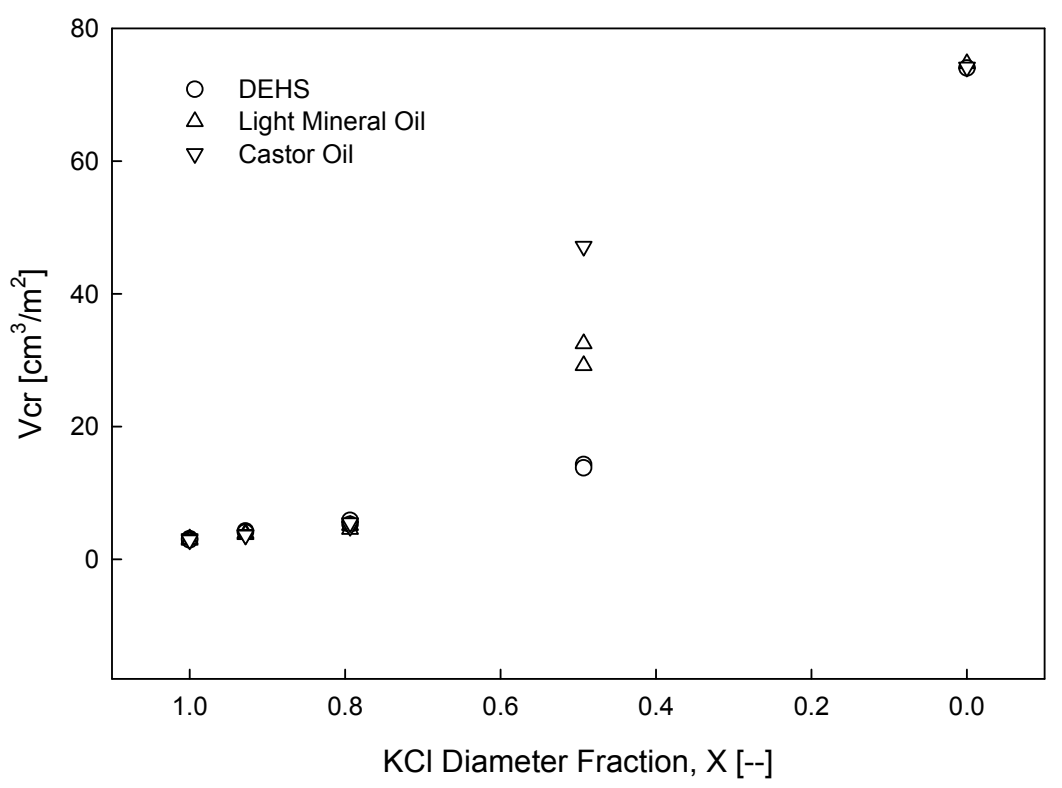

Fig. 3. Critical volume $\left(V_{c r}\right)$ of different coating liquids vs. the core diameter fraction for the glass-fiber filter.

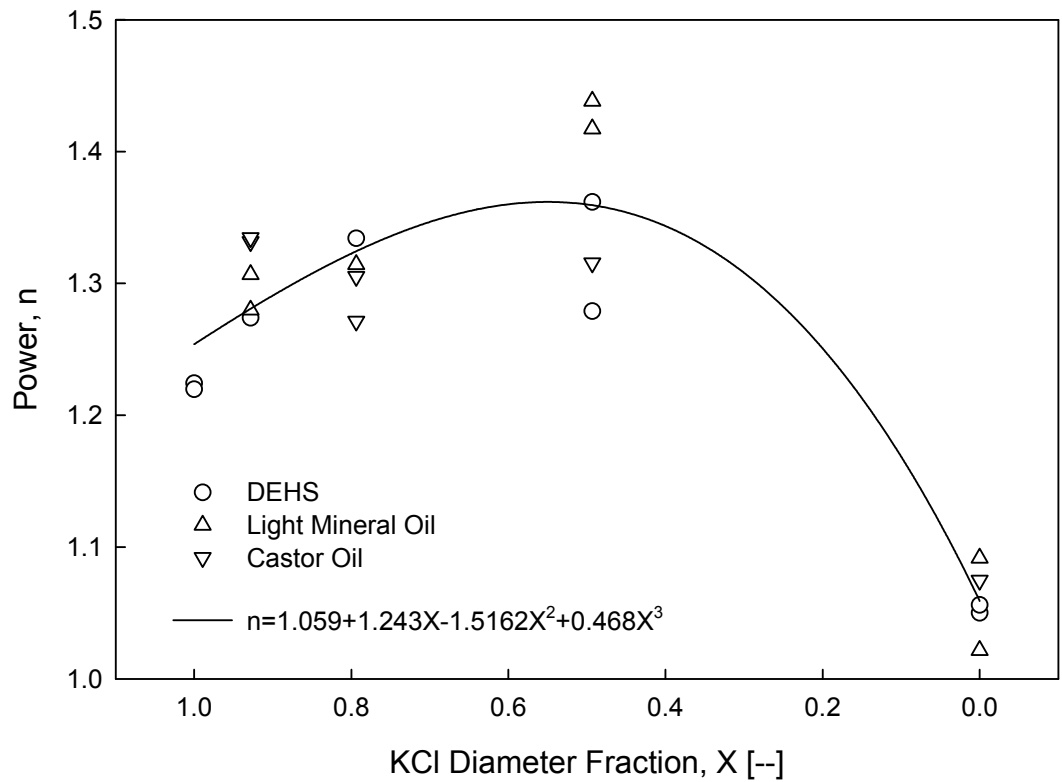

Fig. 4. Exponent $(n)$ of different coating liquids vs. the core diameter fraction for the glass-fiber filter.

For cellulose filter media, the variations of $V_{c r}$ and $n$ values as a function of solid-core diameter fraction were rather complex (Figs. 5 and 6). It is because not only could coating liquid flow over the fiber surfaces of cellulose media, but it could also be absorbed by fibers (Hsiao and Chen, 2015). Moreover, the viscosity of coating liquid completely affected the relative position of the loading curves in a range of solid core diameter fractions. To include the viscosity effect in the filter pressure drop model, Table Curve $3 \mathrm{D}^{\mathrm{TM}}$ was used to establish the relationship among $V_{c r}, n$, liquid viscosity, and solid-core diameter fraction of test particles in the cases with cellulose media. Note that the same procedure was also applied to the parameter $V_{c r}$ in the cases with glass-fiber filter media.
In the analysis, we normalized the values of $V_{c r}$ for different liquids when filter media was loaded with pure liquid particles, and normalized the viscosity of coating liquids, $\mu_{\text {liq }}$, by the water viscosity, $\mu_{w}$. As shown in Figs. $7-$ 9 , all three sets of data can be fitted by a polynomial equation, Eq. (19). The parameters included in Eq. (19) are listed in Table 3. In general, the values of $V_{c r}$ and $n$ can be obtained from Eqs. (18) and (19) for glass-fiber and cellulose filter media loaded with particles coated with oil liquids.

By Eqs. (17)-(19), the pressure drop evolution curve of glass-fiber and cellulose filter media can be calculated when loaded with oil-coated particles having more than $50 \%$ percentage in liquid (i.e., if the solid -core diameter fraction is less than 0.79 ): 


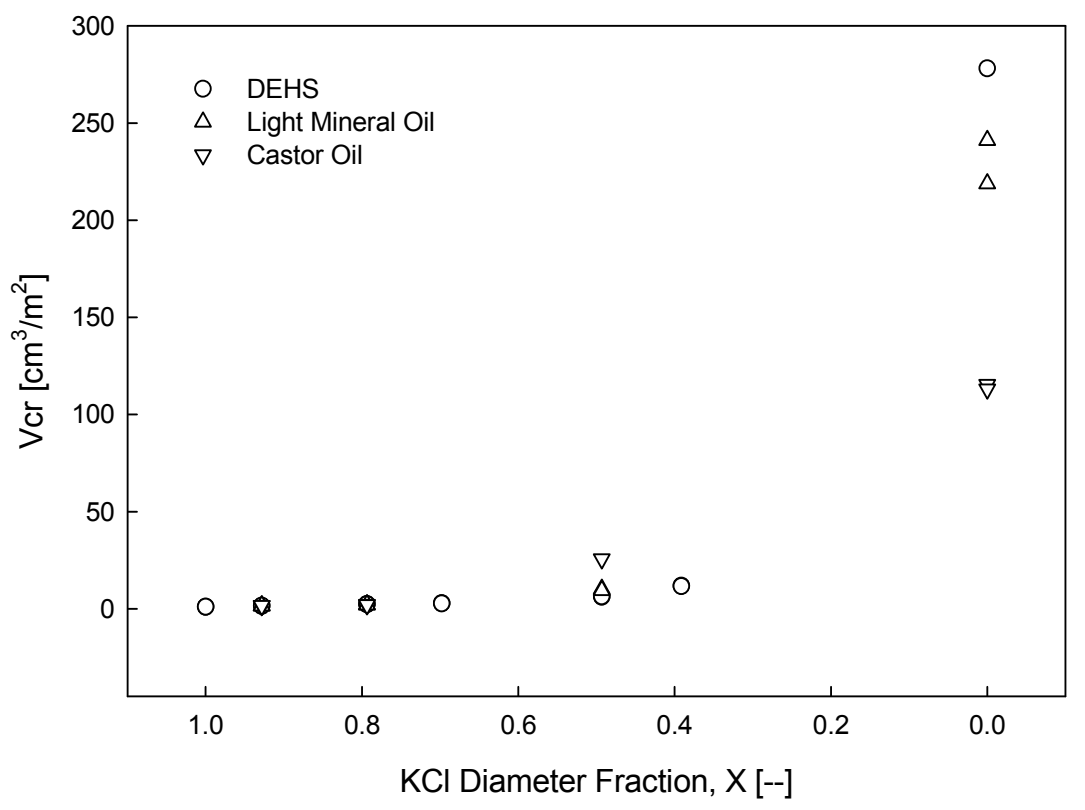

Fig. 5. Critical volume $\left(V_{c r}\right)$ of different coating liquids vs. the core diameter fraction for the cellulose filter.

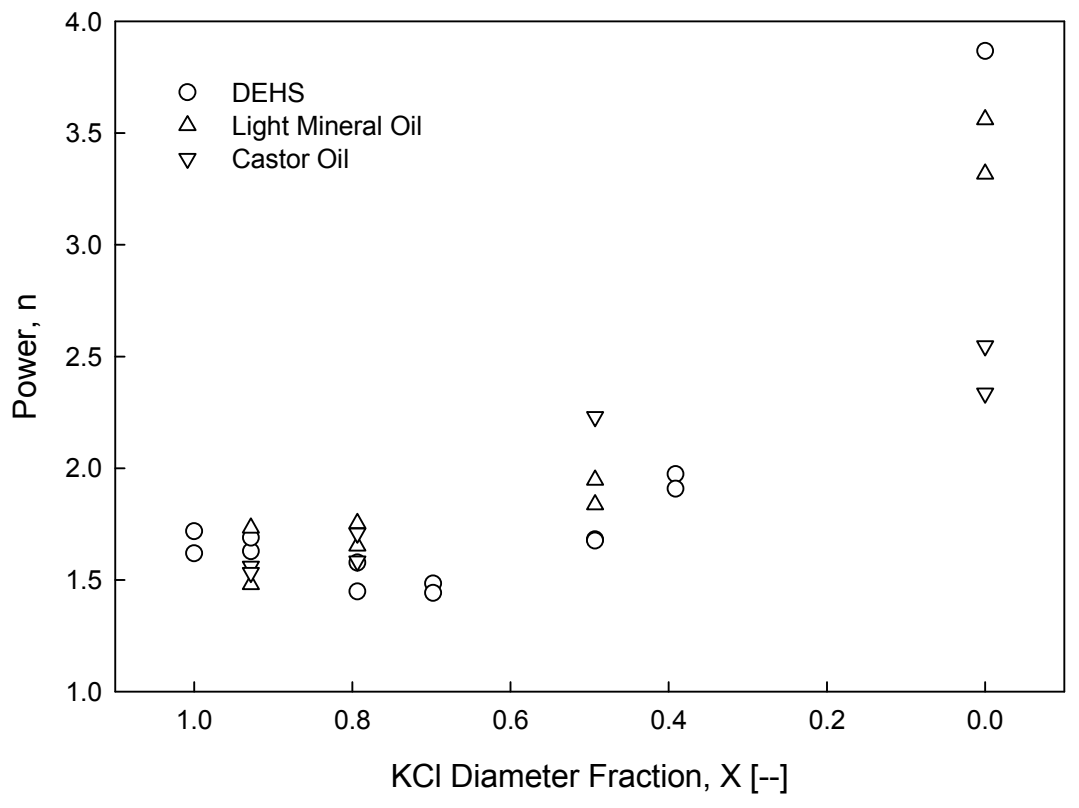

Fig. 6. Exponent $(n)$ of different coating liquids vs. the core diameter fraction for the cellulose filter.

$$
\begin{aligned}
\frac{V_{c r}}{V_{c r, l i q}} \text { or } n & =A+B \cdot X+\frac{C}{\log \left(\mu_{l i q} / \mu_{w}\right)}+D \cdot X^{2} \\
& +\frac{E}{\left[\log \left(\mu_{l i q} / \mu_{w}\right)\right]^{2}}+\frac{F \cdot X}{\log \left(\mu_{l i q} / \mu_{w}\right)}+G \cdot X^{3} \\
& +\frac{H}{\left[\log \left(\mu_{l i q} / \mu_{w}\right)\right]^{3}}+\frac{I \cdot X}{\left[\log \left(\mu_{l i q} / \mu_{w}\right)\right]^{2}} \\
& +\frac{J \cdot X^{2}}{\log \left(\mu_{l i q} / \mu_{w}\right)}
\end{aligned}
$$

for $0<X<0.79$ where $A, B, C, D, E, F, G, H, I$, and $J$ are fitting constants.

\section{CONCLUSION}

The oil-coated particles (i.e., greasy particles) are generally found in machining factories and co-emitted by fuel combustion sources and required to be filtered. When filter media is loaded with such particles, the buildup rate of the filter pressure drop decreases, and the transitional point of the loading pressure drop curve shifts to the heavily loaded mass regime as the oil volumetric percentage of the coated particles rises. In this work, we developed an empirical model to describe the pressure drop curves 


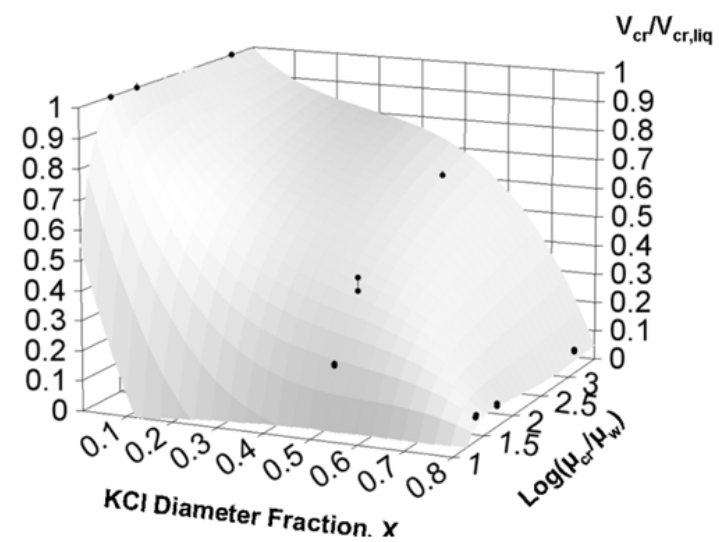

Fig. 7. Fitting result for $V_{c r}$ with the glass-fiber filter.

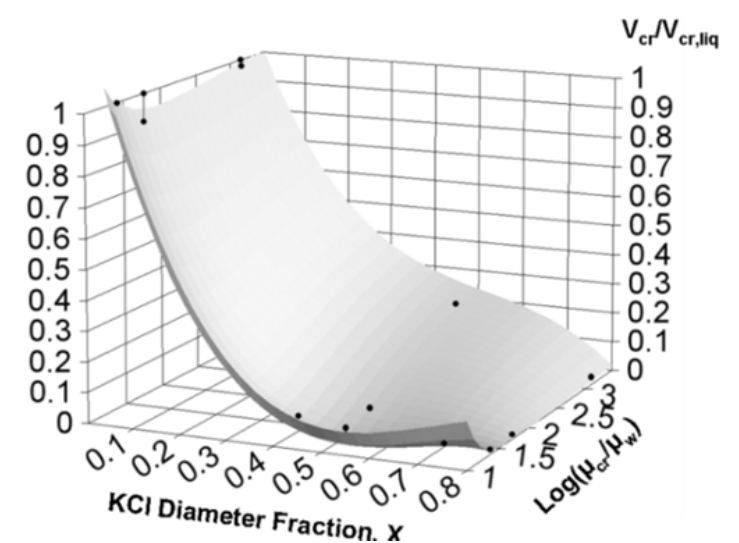

Fig. 8. Fitting result for $V_{c r}$ with the cellulose filter.

(specifically, the filter pressure drop as a function of loaded particle volume per filter media unit area) for filter media loaded with particles coated with oil layers of various thicknesses.

To fit our experimental observations of distinct loading curve characteristics above and below a liquid volumetric percentage of $50 \%$, the model consisted of two parts. When the particles possessed a liquid volumetric percentage of less than $50 \%$, the Bergman model for solid-particle loading was modified to predict a filter pressure drop that increased up to 4 times of the initial pressure drop. In this part of the modeling, a loaded filter was assumed to have two layers: one layer to collect all of the oil-coated particles and the other to remain as a clean medium. The thickness of the first layer can be measured from SEM

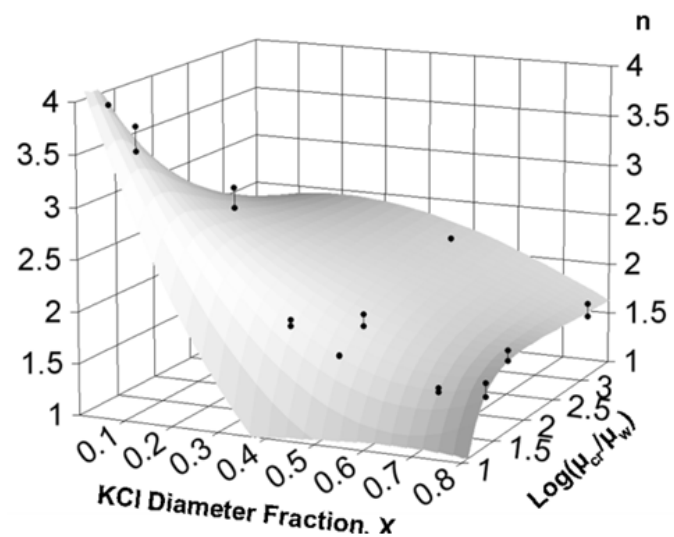

Fig. 9. Fitting result for $n$ with the cellulose filter.

images. The total pressure drop of the loaded filter media was then assumed to be the summation of the layer pressure drops.

When the particles possessed a liquid volumetric percentage of greater than $50 \%$, a power-law equation with two parameters, viz., the exponent $(n)$ and the critical volume $\left(V_{c r}\right)$, was introduced to fit the experimental data. The correlations of the above parameters with the solid-core diameter fraction of particles and the viscosity of the coating oil were established. The overall pressure drop of filter media loaded with oil-coated particles can thus be estimated. However, because the effects of surface tension were not included in the experimental data on which this model is based, the proposed equations (Eqs. (17)-(19)) are applicable only when the coating oil has low surface tension $\left(<35 \mathrm{mN} \mathrm{m}^{-1}\right)$.

\section{NOMENCLATURE}

$\rho \quad$ Fluid density

$U \quad$ Fluid velocity

$d_{f} \quad$ Fiber diameter

$d_{f m} \quad$ Diameter of a dust-loaded fiber

$d_{f, w} \quad$ Coated fiber diameter

$\mu \quad$ Fluid viscosity

$\mu_{\text {liq }} \quad$ Viscosity of coating liquids

$\mu_{w} \quad$ Water viscosity

$\gamma_{L V} \quad$ Liquid surface tension

$f \quad$ Drag coefficient

$F_{D} \quad$ Fiber drag force per unit of fiber length

$F_{l} \quad$ Total drag forces acting on the dust-loaded fibers

Table 3. Fitting constants for different $V_{c r}$ and $n$.

\begin{tabular}{cllll}
\hline$V_{c r} /$ Glass-fiber Filter & & $\mathrm{r}^{2}=0.9976$ & & \\
$A=1.202$ & $B=5.492 \mathrm{E}-1$ & $C=-1.638$ & $D=1.227$ & $E=4.072$ \\
$F=-6.653$ & $G=-4.107$ & $H=-3.089$ & $I=5.015 \mathrm{E}-1$ & $J=7.760$ \\
$V_{c r} /$ Cellulose Filter & & $\mathrm{r}^{2}=0.9983$ & & \\
$A=1.216$ & $B=3.135$ & $C=-8.778 \mathrm{E}-1$ & $D=5.269$ & $E=2.985 \mathrm{E}-1$ \\
$F=-1.508$ & $G=-4.042$ & $H=7.430 \mathrm{E}-1$ & $I=-1.354$ & $J .543$ \\
$n /$ Cellulose Filter & & $\mathrm{r}^{2}=0.9906$ & & \\
$A=1.330$ & $B=6.455$ & $C=2.714$ & $D=-8.783$ & $J .631$ \\
$F=-1.743 \mathrm{E}+1$ & $G=1.259$ & $H=-3.309$ & $I=-1.480$ & $J=1.748 \mathrm{E}+1$ \\
\hline
\end{tabular}


$L_{f} \quad$ Total fiber length per unit area of filter media

$L_{p} \quad$ Total fiber length of newly formed dendrite per unit area of filter media

$Z \quad$ Thickness of the filter

$Z_{f L} \quad$ Thickness of the front (loaded) layer

$\alpha_{f} \quad$ Filter packing density

$\alpha_{p} \quad$ Packing density due to newly formed particle dendrites

$\alpha_{e} \quad$ Packing density at steady-state saturation condition (Raynor and Leith, 2000)

$\alpha_{l} \quad$ Packing density due to perfectly coated liquid on filter fibers

$\alpha_{\text {tube }}$ Maximal value due to the liquid coating in the filter

$\alpha_{f i m} \quad$ Maximal liquid packing density

$\Delta P \quad$ Total pressure drop

$\Delta P_{0} \quad$ Pressure drop of a clean filter

$\Delta P_{l} \quad$ Pressure drop across a dust-loaded filter

$\Delta P_{0}{ }^{\prime} \quad$ Pressure drop of a clean filter layer

$\Delta P_{l}^{\prime} \quad$ Pressure drop across a dust-loaded filter layer

$\Delta P_{s} \quad$ Filter pressure drop at the final steady-state stage

$C_{d m} \quad$ Drag coefficient of dust-loaded fibers per unit filtration area

$V_{\text {ac }}$ Dimensionless accumulated particle volume

$V_{c r} \quad$ Critical volume

$V_{c r, l i q} \quad V_{c r}$ for filter media loaded with pure liquid

$\theta \quad$ Contact angle between a deposited droplet and a fiber

$S_{e} \quad$ Steady-state saturation ratio

$\mathrm{Ca}$ Capillary number (dimensionless)

Bo Bond number (dimensionless)

Dr Drainage rate (dimensionless)

$X \quad$ Diameter fraction of solid core in oil-coated particles

\section{ACKNOWLEDGEMENT}

This research was financially supported by National Taiwan University from Excellence Research Program Core Consortiums (NTUCCP-107L891310) within the framework of the Higher Education Sprout Project by the Ministry of Education (MOE) in Taiwan and partially supported by the Center for Filtration Research at the University of Minnesota. This manuscript was edited by Wallace Academic Editing.

\section{REFERENCES}

Agranovski, I.E. and Braddock, R.D. (1998a). Filtration of liquid aerosols on wettable fibrous filters. AIChE J. 44: 2775-2783.

Agranovski, I.E. and Braddock, R.D. (1998b). Filtration of liquid aerosols on nonwettable fibrous filters. AIChE J. 44: 2784-2790.

Bao, B., He, W., Zhao, H., Xu, B. and Lin, Z. (2015). Modeling penetration through fibrous filter during dynamic filtration. Aerosol Air Qual. Res. 15: 648-656.

Bergman, W., Taylor, R., Miller, H., Bierman, A., Hebard, H., Daroza, R. and Lum, B. (1978). Enhanced filtration program at LLL. A progress report, California Univ.,
Livermore (USA). Lawrence Livermore Lab.

Brown, R.C. (1993). Air filtration: An integrated approach to the theory and applications of fibrous filters. Pergamon, Oxford.

Chang, C., Ji, Z. and Zeng, F. (2016). The effect of a drainage layer on filtration performance of coalescing filters. Sep. Purif. Technol. 170: 370-376.

Chen, C.C., Chen, W.Y., Huang, S.H., Lin, W.Y., Kuo, Y.M. and Jeng, F.T. (2001). Experimental study on the loading characteristics of needlefelt filters with micrometer-sized monodisperse aerosols. Aerosol Sci. Technol. 34: 262-273.

Choi, H.J., Kumita, M., Hayashi, S., Yuasa, H., Kamiyama, M., Seto, T., Tsai, C.J. and Otani, Y. (2017). Filtration properties of nanofiber/microfiber mixed filter and prediction of its performance. Aerosol Air Qual. Res. 17: 1052-1062.

Davies, C. (1953). The separation of airborne dust and particles. Proc. Inst. Mech. Eng. Part B 1: 185-213.

Emi, H., Wang, C.S. and Tien, C. (1982). Transient behavior of aerosol filtration in model filters. AIChE J. 28: 397-405.

Endo, Y., Chen, D.R. and Pui, D.Y.H. (1998). Effects of particle polydispersity and shape factor during dust cake loading on air filters. Powder Technol. 98: 241-249.

Frising, T., Thomas, D., Bémer, D. and Contal, P. (2005). Clogging of fibrous filters by liquid aerosol particles: Experimental and phenomenological modelling study. Chem. Eng. Sci. 60: 2751-2762.

Fuchs, N. and Stechkina, I. (1963). A note on the theory of fibrous aerosol filters. Ann. Occup. Hyg. 6: 27-30.

Gonsalves, V. (1950). A critical investigation on the viscose filtration process. Recl. Trav. Chim. Pays-Bas 69: 873903.

Happel, J. (1959). Viscous flow relative to arrays of cylinders. AIChE J. 5:174-177.

Hermans, P. (1936). Principles of the mathematical treatment of constant-pressure filtration. J. Soc. Chem. Ind. 55: 1-4.

Hsiao, T.C. and Chen, D.R. (2015). Experimental observations of the transition pressure drop characteristics of fibrous filters loaded with oil-coated particles. Sep. Purif. Technol. 149: 47-54.

Jackson, G.W. and James, D.F. (1986). The permeability of fibrous porous media. Can. J. Chem. Eng. 64: 364374.

Japuntich, D.A., Stenhouse, J.I.T. and Liu, B.Y.H. (1994). Experimental results of solid monodisperse particle clogging of fibrous filters. J. Aerosol Sci. 25: 385-393.

Joubert, A., Laborde, J.C., Bouilloux, L., Chazelet, S. and Thomas, D. (2011). Modelling the pressure drop across HEPA filters during cake filtration in the presence of humidity. Chem. Eng. J. 166: 616-623.

Kanaoka, C. and Hiragi, S. (1990). Pressure drop of air filter with dust load. J. Aerosol Sci. 21: 127133-131137.

Kuwabara, S. (1959). The forces experienced by randomly distributed parallel circular cylinders or spheres in a viscous flow at small Reynolds numbers. J. Phys. Soc. Jpn. 14: 527-532. 
Leung, W.W.F. and Hung, C.H. (2008). Investigation on pressure drop evolution of fibrous filter operating in aerodynamic slip regime under continuous loading of sub-micron aerosols. Sep. Purif. Technol. 63: 691-700.

Liew, T.P. and Conder, J.R. (1985). Fine mist filtration by wet filters-I. Liquid saturation and flow resistance of fibrous filters. J. Aerosol Sci. 16: 497-509.

Müller, L., Comte, P., Czerwinski, J., Kasper, M., Mayer, A.C., Gehr, P., Burtscher, H., Morin, J.P., Konstandopoulos, A. and Rothen-Rutishauser, B. (2010). New exposure system to evaluate the toxicity of (scooter) exhaust emissions in lung cells in vitro. Environ. Sci. Technol. 44: 2632-2638.

Mullins, B.J. and Kasper, G. (2006). Comment on: "Clogging of fibrous filters by liquid aerosol particles: Experimental and phenomenological modelling study" by Frising et al. Chem. Eng. Sci. 61: 6223-6227.

Park, B.H., Kim, S.B., Jo, Y.M. and Lee, M.H. (2012). Filtration characteristics of fine particulate matters in a PTFE/glass composite bag filter. Aerosol Air Qual. Res. 12: $1030-1036$.

Raynor, P.C. and Leith, D. (2000). The influence of accumulated liquid on fibrous filter performance. $J$. Aerosol Sci. 31: 19-34.

Sachinidou, P., Bahk, Y.K., Tang, M., Zhang, N., Chen, S.S., Pui, D.Y., Lima, B.A., Bosco, G., Tronville, P. and Mosimann, T. (2017). Inter-laboratory validation of the method to determine the filtration efficiency for airborne particles in the 3-500 $\mathrm{nm}$ range and results sensitivity analysis. Aerosol Air Qual. Res. 17: 2669-2680.

Saleh, A. and Tafreshi, H.V. (2014). A simple seminumerical model for designing pleated air filters under dust loading. Sep. Purif. Technol. 137: 94-108.

Saleh, A.M. and Vahedi Tafreshi, H. (2015). On the filtration performance of dust-loaded trilobal fibers. Sep.
Purif. Technol. 149: 295-307.

Song, C.B., Park, H.S. and Lee, K.W. (2006). Experimental study of filter clogging with monodisperse PSL particles. Powder Technol. 163: 152-159.

Thomas, D., Contal, P., Renaudin, V., Penicot, P., Leclerc, D. and Vendel, J. (1999). Modelling pressure drop in HEPA filters during dynamic filtration. J. Aerosol Sci. 30: 235-246.

Thomas, D., Penicot, P., Contal, P., Leclerc, D. and Vendel, J. (2001). Clogging of fibrous filters by solid aerosol particles experimental and modelling study. Chem. Eng. Sci. 56: 3549-3561.

Veerapaneni, S. and Wiesner, M.R. (1997). Deposit morphology and head loss development in porous media. Environ. Sci. Technol. 31: 2738-2744.

Wei, X., Chen, F., Wang, H., Zhou, H., Ji, Z. and Lin, T. (2017). Efficient removal of aerosol oil-mists using superoleophobic filters. J. Mater. Chem. A 6: 871-877.

Yamada, S., Seto, T. and Otani, Y. (2011). Influence of filter inhomogeneity on air filtration of nanoparticles. Aerosol Air Qual. Res. 11: 155-160.

Zhang, J., Pan, W., Long, Z., Wang, C. and Feng, Z. (2017). Study of the oil mist filtration performance: Pressure drop characteristics and filter efficiency model. Aerosol Air Qual. Res. 17: 1063-1072.

Zhou, B., Xu, Y., Fan, J.Q., Chen, L.P., Li, F. and Xue, K. (2017). Numerical simulation and experimental validation for the filtration performance of fibrous air filter media with LB method. Aerosol Air Qual. Res. 17: 2645-2658.

Received for review, January 1, 2019 Revised, April 29, 2019 Accepted, June 1, 2019 